\title{
Pre-operative fasting times for clear liquids at a tertiary children's hospital; what can be improved?
}

Received March 16, 2021

Revised May 5, 2021

Accepted May 10, 2021

\section{Corresponding author}

Alexander R. Schmidt, M.D. Department of Anesthesiology, Perioperative and Pain Medicine, Division of Pediatric Anesthesia, Lucile Packard Children's Hospital, Stanford University School of Medicine, 300 Pasteur Drive, Stanford, CA 94305-5640, USA

Tel: 1-650-497-8000

Fax: 1-650-725-8544

E-mail: scale@stanford.edu

\section{Alexander R. Schmidt, James Fehr, Janice Man, Genevieve D’Souza, Ellen Wang, Rebecca Claure, and Julianne Mendoza}

Department of Anesthesiology, Perioperative and Pain Medicine, Stanford University School of Medicine, Stanford, CA, USA

Background: The goal of preoperative fasting is to prevent pulmonary aspiration during general anesthesia. Fasting times are often prolonged leading to patient discomfort and risk for adverse events. This retrospective quality improvement survey evaluated effective nil-per-os (NPO) times and causes for prolonged NPO times with the aim to suggest improvement strategies by a newly founded fasting task force.

Methods: Data from all electronic anesthesia records from 2019 at our institution were reviewed for fasting times. Our NPO instructions follow American Society of Anesthesiology guidelines and are calculated based on the patient's arrival time ( $90 \mathrm{~min}$ before operating room [OR] time). Primary outcome was the effective NPO time for clear liquids, secondary outcomes were incidence of delays and the parental compliance with the NPO instructions. Data are presented as median (interquartile range).

Results: In total 9,625 cases were included in the analysis. NPO time was documented in $72.1 \%$ with a median effective NPO time of 7:13 $\mathrm{h}(7: 36)$. OR in room times were documented in 72.8\%, 2,075 (29.5\%; median time 0:10 h [0:21]) were earlier and 4,939 (70.5\%; median time 0:29 h [0:54]) were later than scheduled. Parental NPO compliance showed a median deviation for clear liquid intake of 0:55 h (8:30).

Conclusions: This study revealed that effective NPO times were longer than current ASA guidelines. Contributing causes include case delays and parental non-compliance to NPO instructions. Thus, task force recommendations include change NPO instruction calculations to scheduled OR time versus arrival time, and encourage parents to give their child clear liquids at the instructed time.

Keywords: Anesthesiology; Fasting; Quality improvement; Surveys and questionnaires.

\section{INTRODUCTION}

In elective cases, preoperative fasting is intended to prevent pulmonary aspiration of gastric contents during general anesthesia. The American Society of Anesthesiology (ASA) guidelines suggest $2 / 4 / 6 \mathrm{~h}$ for fasting for clear liquids, breast- milk and light meals, respectively [1]. It is well known that the fasting times are often prolonged due to organizational issues, communication problems, or because children do not receive clear liquids up until the cutoff time for various reasons such as not wanting to wake the child for a drink if they are scheduled early in the morning [2-5]. Prolonged

This is an Open Access article distributed under the terms of the Creative Commons Attribution Non-Commercial License (http://creativecommons.org/licenses/by-nc/4.0) which permits unrestricted non-commercial use, distribution, and reproduction in any medium, provided the original work is properly cited.

Copyright (C) the Korean Society of Anesthesiologists, 2021 
fasting may lead to hypovolemia and disequilibrium of the metabolic status putting the patient at risk for adverse events including arterial hypotension, difficult intravenous access, or hypoglycemia [6,7]. Isserman et al. [8] and Newton et al. [9] reported in their quality improvement studies methods for optimization in timing of clear liquid fasting. Both studies used the percentage of patients having clear liquids within four hours prior to anesthesia as the improvement criteria. Isserman et al. [8] showed an increase from 20 to $63 \%$ and Newton et al. [9] from 19 to $72 \%$ after implementing new "nil-per-os" (NPO) instructions allowing more liberal clear liquid fasting for one hour prior to anesthesia. This allowed them to offer eligible children a drink on arrival and reduced their fasting times significantly. This step of reducing the fasting time for clear liquids to one hour was justified by the current evidence showing that one hour of clear liquid fasting is not associated with increased incidence of aspiration [10-14]. Presently, this regimen is corroborated by recently published consensus statements from international anesthesia societies recommending clear liquid intake up to one hour prior to anesthesia [15-18]. However, in the United States, nationwide anesthesia departments follow the current ASA guidelines on clear liquid fasting of two hours prior to anesthesia. Hence, the Isserman et al. [8] and Newton et al. [9] published improvement strategies have not been widely implemented. Thus, other solutions to achieve an optimized clear liquid fasting of two hours with the aim to minimize the negative effects of prolonged fasting need to be described.

The aim of this retrospective quality improvement survey was to evaluate the current effective clear liquid fasting times at our institution, to further investigate causes for prolonged NPO hours and to suggest options to decrease barriers to adherence of current ASA guidelines.

\section{MATERIALS AND METHODS}

After approval from the Institutional Review Board at Stanford University School of Medicine (no. IRB-56152) all electronic medical anesthesia records of the year 2019 at the Division of Pediatric Anesthesia of Lucile Packard Children's Hospital (LPCH, Stanford University School of Medicine, Stanford, CA, USA) were reviewed. Emergent cases were excluded for statistical analysis. All inpatients were also excluded in the statistical analysis due to variability in NPO instructions (NPO from midnight for all liquids and solids to allow more flexibility for scheduling during the next day), which were studied and improved after a quality improvement study published by Nye et al. [19] in 2018.

At our institution patients are instructed to arrive to the hospital at least 90 min prior to their scheduled surgical start time. The NPO instructions are in accordance with the current ASA guidelines and are calculated based on the patient's arrival time (see Fig. 1).

To investigate and discuss the findings of this retrospective analysis and to further create an improvement plan, a task force was founded. This task force consisted of the chief of the pediatric anesthesia division (JF), a member of the anesthesia quality improvement team (JM), the OR Medical Director (RC), two members of the Pediatric Anesthesia Resource Center team (JM and GD) and an expert on preoperative fasting (ARS).

The primary outcome was to measure the effective NPO time for clear liquid, defined as time from last intake of clear liquids to "in room time". Secondary outcomes were the incidence of "in room time" compared to scheduled start time and the parental compliance with the NPO instructions (defined as time between instructed and actual NPO time) for patients being allowed to drink clear liquid after $7 \mathrm{AM}$ on their day of surgery.

In addition, all pediatric anesthesia attendings at LPCH were asked to participate in an anonymous online survey to evaluate their willingness to deviate from the existing ASA guidelines for clear liquid fasting. They were asked if they would induce anesthesia (intravenous and mask, respectively) in a child that was fasted for clear liquids for 60 , 90 , or $105 \mathrm{~min}$.

Data were extracted from our electronic health record (EHR) system, Epic (November 2019 version, Epic Systems, Inc., USA) to Excel (Microsoft Excel, edition 2016, Microsoft Corp., USA) and compiled in SPSS (IBM SPSS statistics, version 23, IBM Co., USA) for statistical analysis. Data are presents as median (interquartile range; minimum-maximum) or count and percent were appropriate. Times are displayed as hh:mm (e.g., 1:46 h = $1 \mathrm{~h}$ and $46 \mathrm{~min}$ ).

A first screening of the data showed very few cases with NPO times under $2 \mathrm{~h}(\mathrm{n}=88)$ and some cases with NPO times over 18:00 $\mathrm{h}(\mathrm{n}=148)$. This data was validated independently by two authors (ARS and JM). If both reviewers found an error with documentation (e.g., last time for clear fluid intake documented as PM instead of AM), the case was excluded for further analysis. Otherwise, all other cases were included for analysis. 


\section{Stanford Children's Health Lucile Packard
Children's Hospital
Stanford \\ NPO - No Eating or Drinking Guidelines Before Surgery}

\section{www.stanfordchildrens.org/en/child-visit/npo-guidelines}

Our general NPO (no eating or drinking) instructions are listed below. These are enforced to keep your child as safe as possible. If these guidelines are not followed, your child's procedure or surgery may be delayed or cancelled. A small number of children will receive special guidelines that differ from these. Follow the instructions given by your anesthesia provider.

1. Stop food and candy at midnight. Food includes anything that's not formula, milk, breast milk or clear liquids.

2. Stop formula and milk 6 hours prior to arrival time.

3. Stop breast milk 4 hours prior to arrival time.

4. Stop all clear liquids 2 hours prior to arrival time. Clear liquids include only water, clear apple juice (no pulp, no apple cider), Pedialyte and Gatorade.
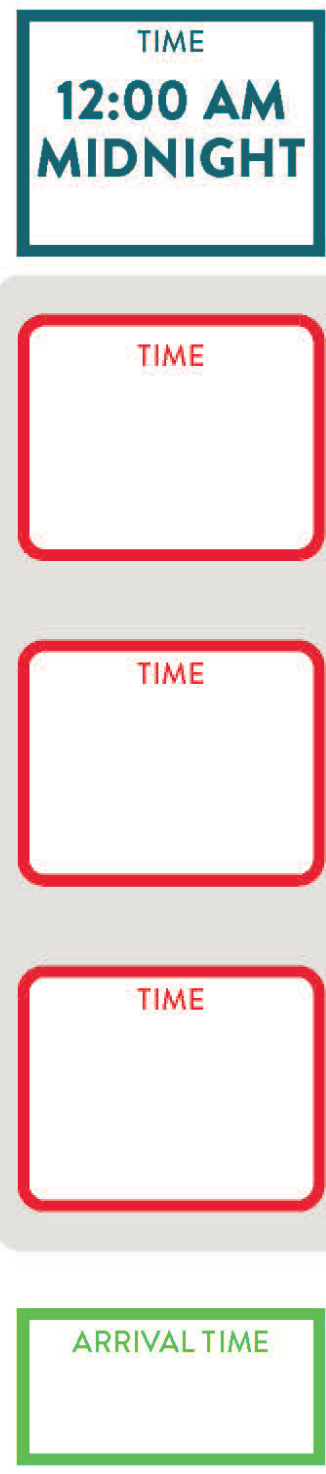

6) Stanford/MEDICINE

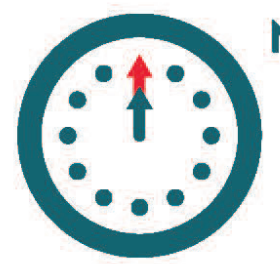

NIGHT BEFORE SURGERY

STOP all food and candy

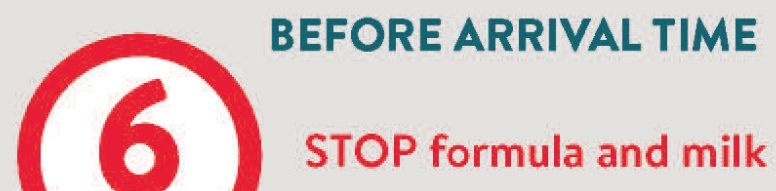

STOP breast milk

STOP all clear liquids

HOURS

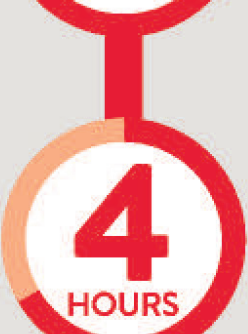

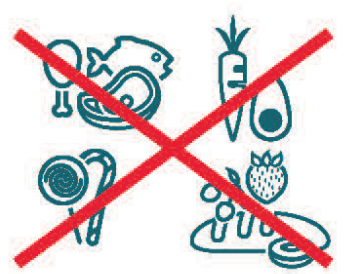
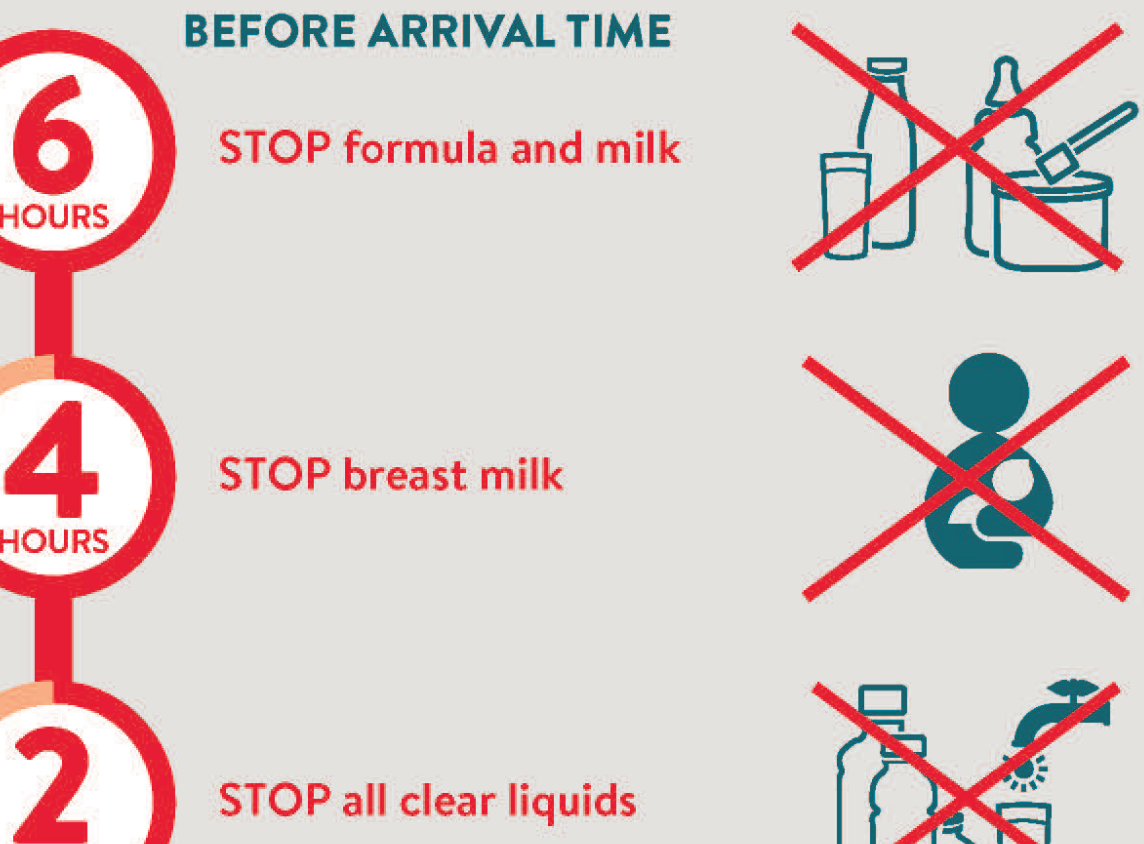

HOURS
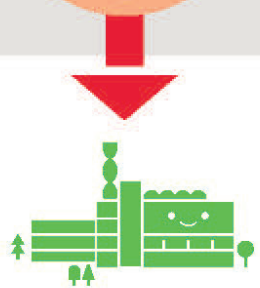

Arrival

Day

Date

Q Plesse recycle.
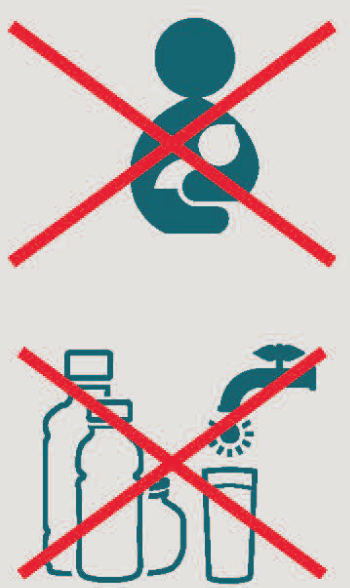

Fig. 1. Displayed is the current nil-per-os (NPO) instruction for our patients based on arrival time. The written text includes the statement the case might be "delayed or cancelled" if the instructions are not followed. NPO for food and candy is always from midnight. 


\section{RESULTS}

\section{NPO data}

A total of 12,623 anesthesia records were extracted from our EHR. From these 540 emergent cases and 2,347 inpatients were excluded prior to analysis. After data validation, 48 cases with NPO times under two hours and 63 cases over $18 \mathrm{~h}$ were excluded. Of the remaining 9,625 cases, $6,841(71.1 \%)$ were ambulatory patients and 2,784 (28.9\%) were outpatients admitted postoperatively. Patients' mean age was 8.3 years $(10.3 ; 0.0-68.7)$ and ASA classifications were as follows: 2,146 (22.3\%) ASA-1, 3,929 (40.8\%) ASA-2, 3,427 (35.6\%) ASA-3, 119 (1.2\%) ASA-4 and four missing ASA documentations.

Arrival time to hospital was documented in 9,054 cases (94.1\%) and the mean time from arrival to the hospital until effective "in room time" was 1:46 h (1:01; 0:14-15:57 h), with $5.1 \%$ of the patients being in the OR less than one hour after arrival and $60.4 \%$ being in the OR less than two hours after arrival.

Data for the primary outcome "effective NPO time for clear liquids" were documented in 6,940 cases $(72.1 \%)$. The median effective NPO was 7:13 h (7:36; 1:36-23:38 h). For 7,067 patients $(73.4 \%)$ the instructed NPO times were documented with a median of $3: 20 \mathrm{~h}(0: 05 ; 2: 00-23: 55 \mathrm{~h})$. In $7,014(72.8 \%)$ cases the scheduled "in room time" was documented, 2,075 (29.5\%) were in the operating room (OR) earlier than scheduled with a median time earlier of $0: 10 \mathrm{~h}$ $(0: 21 ; 0: 01-4: 37 \mathrm{~h})$ and 4,939 (70.5\%) were in the OR later than scheduled with a median time of $0: 29 \mathrm{~h}(0: 54 ; 0: 00$ 11:06 h).

The number of patients allowed to drink clear liquids after 7 AM until their instructed NPO time was 3,084 patients. The median instructed NPO time for clear liquids was 3:20 h $(0: 10 ; 2: 00-23: 55 \mathrm{~h})$ and the median effective NPO time was 5:14 h (7:39; 1:43-23:38 h). Parental NPO compliance (as defined in the methods section) was calculated and showed a median deviation of clear liquid intake of 0:55 h (8:30; 0:0018:30 h). A detailed analysis revealed $42 \%$ of patients drank clear liquids within $0: 30 \mathrm{~h}$ prior to the instructed NPO time, $53.7 \%$ within $1: 00 \mathrm{~h}$ prior and $64.3 \%$ within $2: 00 \mathrm{~h}$ prior. Over $30 \%$ of the patients allowed to drink clear liquids after $7 \mathrm{AM}$ on the day of surgery had an NPO time for clear liquids longer than 4:00 h.

\section{Online survey}

The response rate for the anonymous online survey was $43.9 \%$ (25 out of 57 pediatric anesthesia attendings). The majority of anesthesiologists were willing to induce anesthesia via mask ( $72 \%)$ in children who were NPO for clear liquids for at least $105 \mathrm{~min}$ and intravenously (84\%) if they were NPO for clear liquids for at least $90 \mathrm{~min}$.

Based on the fact that the majority of attendings would be willing to induce anesthesia (both intravenous and mask) 15 min earlier than the recommended ASA 2:00 h (120-105 min $=15 \mathrm{~min}$ ) and the assumption that in most cases the time from "in room" until anesthesia induction is about $10 \mathrm{~min}$, detailed frequencies of earlier "in room time" were investigated. Of the total of 2,075 (29.5\%) cases in the OR earlier than scheduled, $58 \%$ are less than 15 min earlier in the OR, $74.7 \%$ less than 25 min earlier and 3.5\% more than one hour earlier. Based on this data, a change of NPO instructions to "2:00 $\mathrm{h}$ prior to the scheduled in room time" may result in a delayed start time in $5.4 \%$ of all our annual cases (518 cases per year or two cases per day).

\section{DISCUSSION}

This retrospective quality improvement survey investigated the fasting times for clear liquids at a tertiary children's hospital and the causes for prolonged fasting times. The main findings were that the median effective NPO time for clear liquids is excessively prolonged, only a minority of patients come in the OR earlier than scheduled and a majority of patients do not drink clear liquids up to the time they are allowed.

Similar studies in the past have shown prolonged fasting times for clear liquids with similar results to our study [2-5]. Prolonged fasting of clear liquids has been associated with distress for the patient and the parents [20], a higher incidence of irritability and thirst in the pediatric population [21], increased risk of hypotension during anesthesia induction, and a catabolic state [7]. Therefore, every effort should be undertaken to reduce prolonged fasting times for clear liquids.

The present study helped identify various causes for prolonged fasting for clear liquids. First, the NPO instruction are based on arrival time and thus cause a median lengthening in NPO times for clear liquids by 1:20 h. The present study revealed that the majority of cases start with a delay and therefore, this additional time buffer is not justified. As a first step, we strongly recommend NPO instructions based on the scheduled "in room time" and not on the instructed hospital 
arrival time. Opponents might argue with the possibility of causing a delay for patients with an earlier than scheduled "in room time". This argument can be refuted by the following two reasons. First, the incidence of a possible delay for patients going to the OR earlier than scheduled in this study was low (5.4\%). Second and more importantly, is the fact that the theoretical delay is based on the hypothesis that all patients have a fasting time of two hours for clear liquids. Based on the data of this study this will rarely be the case. Even Isserman et al. [8] and Newton et al. [9] still reported mean fasting times around six and three hours respectively after implementation of their new NPO instructions of only one hour for clear liquids. Both these quality improvement studies suggested a more progressive regime allowing intake of clear liquids upon hospital arrival $[8,9]$. Unfortunately, the findings of the present study with $5.1 \%$ of patients in the OR less than one hour after arrival and $60.4 \%$ in OR less than two hours show that allowing liquid intake upon arrival may result in delays at our institution, since we follow the current ASA guidelines recommending two hours for clear liquid fasting.

NPO compliance for patients instructed to drink after 7 AM must be improved. Only 53.7\% patients drank clear liquids within 60 min prior to the instructed NPO time and over $30 \%$ of the patients had a NPO time for clear liquids longer than $4 \mathrm{~h}$. This is in accordance with findings from Best et al. [22] showing that patients often choose to fast longer than instructed. One reason for this, at our institution, is the wording of our NPO instructions stating that "If these guidelines are not followed, your child's procedure or surgery might be delayed or cancelled" (see Fig. 1). The use of punative statements in NPO instructions should be avoided. Thus, we recommend changing our NPO instructions to encourage or even advocate for the intake of clear liquids at the instructed time. Highlighting the benefits of clear liquid intake (less thirst and anxiety, better behavior and more comfort [20,23-25]) to the parents will support their NPO compliance. This cultural change of encouragement for NPO instructions was previously suggested by other quality improvement studies [8,9]. Fig. 2 shows an example of an encouraging NPO instructions suggested by the task force for implementation at our institution.

Finally, the anonymous online survey revealed that about $50 \%$ of the anesthesia attendings would not perform a mask induction on patients who fasted "only" one hour for clear liquids. This practice is in compliance with the current ASA guidelines recommending an NPO time of two hours for clear liquids. Recently published studies showed that one hour of
Please Check-in at at the front desk of LPCH

\section{INSTRUCTIONS FOR FOOD \& LIQUID INTAKE}

Your child will need to be fastened prior to anesthesia. This is necessary so that your child's stomach is empty and to prevent from serious safety risks. But it is also important that your child does not suffer from hunger and thirst. Therefore, please try to give your child something to eat and drink at the times given below. Giving your child something at this time will still be safe.

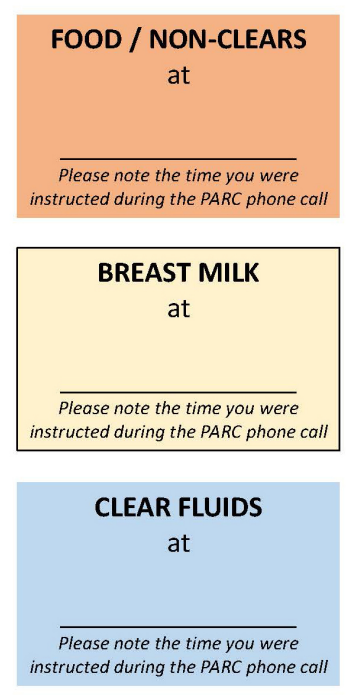

Allowed are: toast with jam, cornflakes with milk, orange juice, chocolate milk, formula milk.

Allowed are:

Water, Gatorade, Pedilyte, tea with no milk, clear apple juice.

Fig. 2. Displayed is an example of a more appropriate nil-peros (NPO) instruction encouraging parents to give their child clear liquids. NPO times are calculated based on the scheduled anesthesia start time. LPCH: Lucile Packard Children's Hospital.

clear liquid fasting is safe [10-14] and have led to publication of several consensus statements by international anesthesia societies recommending clear liquid intake up to one hour prior to anesthesia [15-18]. This dichotomy requires an institutional investigation regarding providers' biases with regard to more liberal liquid fasting. We suggest initiating an anonymous survey to achieve a departmental consensus agreement and to publish a standard operating procedure. This will allow guidance for all anesthesiology providers.

This study has several limitations that need to be addressed. This is a retrospective study from a single center. Other centers might have different NPO instructions and resulting NPO times. Also, at our institution, consecutive cases do not have concurrent anesthesia times. Other institutions may have the ability to induce the next patient in a separate induction room and therefore may need to add additional NPO buffer time to prevent delays. Some of these patients with excessive NPO times may have been placed on intravenous maintenance fluids and therefore, should have been excluded from analysis. The causes for a delayed start (pa- 
tient not ready, anesthesia or surgical delays) were not investigated in detail and also case cancellations for NPO violations were not investigated. Finally, a possible improvement of clear liquid fasting times with the changes could not reported as these changes are recent. Both Isserman et al. [8] and Newton et al. [9] reported a period of over 2 years until improvement was seen. Hence, we thought it important to present the results of our study as it could have implications for other institutions.

Based on the findings of this retrospective quality improvement survey the task force highly recommends the following change in practice at LPCH:

1. Allowing patients to drink clear liquids calculated on the scheduled "in room time".

2. Parents should be advised to give their child something to drink at the instructed time even if they have to wake up their child.

3. Based on the evidence that one-hour clear liquid fasting is safe, attempt to minimize delay anesthesia induction in cases when the NPO time for clear liquid fasting is "only" $105 \mathrm{~min}$.

How to implement in daily clinical routine:

1. Use readily available anesthesia diet orders in the electronic medical record for placing the NPO orders. This will increase adherence and cause less calculation mistakes.

2. With regards to the NPO instructions, we use clear verbiage on our internet website, as well as on the paperwork handed out to families during the pre-anesthesia visit. It is of great importance to go through these instructions in detail with the family and actively encourage them to give fluids up to the allowed time.

3. For patients unable to tolerate prolonged NPO times (e.g., neonates), these cases should be scheduled as first case in the morning to minimize the risk of delayed case start.

4. Investigation of cases with a high incidence of taking longer than scheduled. This will allow more accurate scheduling for the future and decrease the risk of delays for the cases to follow.

In conclusion, we identified three factors that cause prolonged fasting times at our institution. Since prolonged fasting can be associated with hypovolemia and disequilibrium of the metabolic status and thus, puts patients at risk for adverse events including arterial hypotension, difficult intravenous access, or hypoglycemia every effort should be attempted to minimize prolonged fasting times. Our results have led us to the conclusion that our NPO times should be based on the scheduled "in room time", that we should use encouraging instead of punitive language for NPO instructions and that anesthesia staff should not delay anesthesia induction if the fasting time for clear fluids is close to $2 \mathrm{~h}$. Each institution should review their performance on fasting times and identify areas of improvement.

\section{CONFLICTS OF INTEREST}

No potential conflict of interest relevant to this article was reported.

\section{DATA AVAILABILITY STATEMENT}

The datasets generated during and/or analyzed during the current study are not publicly available due (Stanford University Patient Privacy Policy) but are available from the corresponding author on reasonable request.

\section{AUTHOR CONTRIBUTIONS}

Conceptualization: Alexander R. Schmidt, Julianne Mendoza. Data curation: Alexander R. Schmidt, Ellen Wang, Julianne Mendoza. Formal analysis: Alexander R. Schmidt, Janice Man, Genevieve D'Souza, Julianne Mendoza. Methodology: Alexander R. Schmidt, Julianne Mendoza. Project administration: Alexander R. Schmidt, James Fehr, Rebecca Claure. Visualization: Alexander R. Schmidt. Investigation: Alexander R. Schmidt. Resources: James Fehr, Rebecca Claure. Validation: Alexander R. Schmidt, Julianne Mendoza. Writing - original draft: Alexander R. Schmidt. Writing review \& editing: James Fehr, Janice Man, Genevieve D'Souza, Ellen Wang, Rebecca Claure, Julianne Mendoza.

\section{ORCID}

Alexander R. Schmidt, https://orcid.org/0000-0001-7667-0308 James Fehr, https://orcid.org/0000-0002-6914-0226 Janice Man, https://orcid.org/0000-0001-6594-9485 Genevieve D'Souza, https://orcid.org/0000-0003-4801-6215 Ellen Wang, https://orcid.org/0000-0002-9151-938X Rebecca Claure, https://orcid.org/0000-0001-6672-7040 Julianne Mendoza, https://orcid.org/0000-0002-4569-0071

\section{REFERENCES}

1. Practice guidelines for preoperative fasting and the use of pharmacologic agents to reduce the risk of pulmonary aspira- 
tion: application to healthy patients undergoing elective procedures: an updated report by the American Society of Anesthesiologists Task Force on preoperative fasting and the use of pharmacologic agents to reduce the risk of pulmonary aspiration. Anesthesiology 2017; 126: 376-93.

2. Engelhardt T, Wilson G, Horne L, Weiss M, Schmitz A. Are you hungry? Are you thirsty?--fasting times in elective outpatient pediatric patients. Paediatr Anaesth 2011; 21: 964-8.

3. Schmitz A, Kellenberger CJ, Neuhaus D, Schroeter E, Deanovic D, Prüfer F, et al. Fasting times and gastric contents volume in children undergoing deep propofol sedation--an assessment using magnetic resonance imaging. Paediatr Anaesth 2011; 21: 685-90.

4. Arun BG, Korula G. Preoperative fasting in children: an audit and its implications in a tertiary care hospital. J Anaesthesiol Clin Pharmacol 2013; 29: 88-91.

5. Beck CE, Rudolp D, Becke-Jakob K, Schindler E, Etspüler A, Trapp A, et al. Real fasting times and incidence of pulmonary aspiration in children: results of a German prospective multicenter observational study. Paediatr Anaesth 2019; 29: 1040-5.

6. Dennhardt N, Beck C, Huber D, Nickel K, Sander B, Witt LH, et al. Impact of preoperative fasting times on blood glucose concentration, ketone bodies and acid-base balance in children younger than 36 months: a prospective observational study. Eur J Anaesthesiol 2015; 32: 857-61.

7. Dennhardt N, Beck C, Huber D, Sander B, Boehne M, Boethig $\mathrm{D}$, et al. Optimized preoperative fasting times decrease ketone body concentration and stabilize mean arterial blood pressure during induction of anesthesia in children younger than 36 months: a prospective observational cohort study. Paediatr Anaesth 2016; 26: 838-43.

8. Isserman R, Elliott E, Subramanyam R, Kraus B, Sutherland T, Madu C, et al. Quality improvement project to reduce pediatric clear liquid fasting times prior to anesthesia. Paediatr Anaesth 2019; 29: 698-704.

9. Newton RJG, Stuart GM, Willdridge DJ, Thomas M. Using quality improvement methods to reduce clear fluid fasting times in children on a preoperative ward. Paediatr Anaesth 2017; 27: 793-800.

10. Schmitz A, Kellenberger CJ, Liamlahi R, Studhalter M, Weiss M. Gastric emptying after overnight fasting and clear fluid intake: a prospective investigation using serial magnetic resonance imaging in healthy children. Br J Anaesth 2011; 107: 425-9.

11. Schmitz A, Kellenberger CJ, Lochbuehler N, Fruehauf M, Klaghofer R, Fruehauf H, et al. Effect of different quantities of a sugared clear fluid on gastric emptying and residual volume in children: a crossover study using magnetic resonance imaging.
Br J Anaesth 2012; 108: 644-7.

12. Andersson H, Zarén B, Frykholm P. Low incidence of pulmonary aspiration in children allowed intake of clear fluids until called to the operating suite. Paediatr Anaesth 2015; 25: 770-7.

13. Schmidt AR, Buehler P, Seglias L, Stark T, Brotschi B, Renner T, et al. Gastric $\mathrm{pH}$ and residual volume after 1 and $2 \mathrm{~h}$ fasting time for clear fluids in children†. Br J Anaesth 2015; 114: 477-82.

14. Schmidt AR, Buehler KP, Both C, Wiener R, Klaghofer R, Hersberger $\mathrm{M}$, et al. Liberal fluid fasting: impact on gastric $\mathrm{pH}$ and residual volume in healthy children undergoing general anaesthesia for elective surgery. Br J Anaesth 2018; 121: 647-55.

15. Thomas M, Morrison C, Newton R, Schindler E. Consensus statement on clear fluids fasting for elective pediatric general anesthesia. Paediatr Anaesth 2018; 28: 411-4.

16. Rosen D, Gamble J, Matava C; Canadian Pediatric Anesthesia Society Fasting Guidelines Working Group. Canadian Pediatric Anesthesia Society statement on clear fluid fasting for elective pediatric anesthesia. Can J Anaesth 2019; 66: 991-2.

17. Linscott D. SPANZA endorses 1-hour clear fluid fasting consensus statement. Paediatr Anaesth 2019; 29: 292.

18. Disma N, Thomas M, Afshari A, Veyckemans F, De Hert S. Clear fluids fasting for elective paediatric anaesthesia: the European Society of Anaesthesiology consensus statement. Eur J Anaesthesiol 2019; 36: 173-4.

19. Nye A, Conner E, Wang E, Chadwick W, Marquez J, Caruso TJ. A pilot quality improvement project to reduce preoperative fasting duration in pediatric inpatients. Pediatr Qual Saf 2019; 4: e246.

20. Schreiner MS, Triebwasser A, Keon TP. Ingestion of liquids compared with preoperative fasting in pediatric outpatients. Anesthesiology 1990; 72: 593-7.

21. Brady M, Kinn S, Ness V, O’Rourke K, Randhawa N, Stuart P. Preoperative fasting for preventing perioperative complications in children. Cochrane Database Syst Rev 2009; 4: CD005285.

22. Best C, Wolstenholme S, Kimble J, Hitchings H, Gordon HM. How 'nil by mouth' instructions impact on patient behaviour. Nurs Times 2004; 100: 32-4.

23. Splinter WM, Stewart JA, Muir JG. The effect of preoperative apple juice on gastric contents, thirst, and hunger in children. Can J Anaesth 1989; 36: 55-8.

24. Splinter WM, Stewart JA, Muir JG. Large volumes of apple juice preoperatively do not affect gastric $\mathrm{pH}$ and volume in children. Can J Anaesth 1990; 37: 36-9.

25. Castillo-Zamora C, Castillo-Peralta LA, Nava-Ocampo AA. Randomized trial comparing overnight preoperative fasting period vs oral administration of apple juice at 06:00-06:30 am in pediatric orthopedic surgical patients. Paediatr Anaesth 2005; 15: 638-42. 